\title{
INDUCTIVE VOLTAGE ADDER DRIVEN X-RAY SOURCES FOR HYDRODYNAMIC RADIOGRAPHY*
}

\author{
John Maenchen, S. Cordova, J. Gustwiller, D.L. Johnson, P. Menge, \\ I. Molina, C. Olson, S. Rosenthal, and D. Rovang \\ Sandia National Laboratories, PO Box 5800, Albuquerque, New Mexico 87185-1193 \\ B. Oliver and D. Welch \\ Mission Research Corporation, 1720 Randolph Road SE, Albuquerque, New Mexico 87106 \\ V. Bailey and I. Smith \\ Pulsed Sciences Inc., 600 McCormick, San Leandro, California 94577

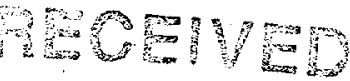 \\ D. Droemer, E. Hunt, G. MacLeod, and L. Woo \\ Bechtel Nevada Corporation, P.O. Box 98521, Las Vegas, Nevada 89193-8521

Abstract

Inductive Voltage Adder (IVA) accelerators were developed to provide high-current (100s of $\mathrm{kA}$ ) power pulses at high voltage (up to $20 \mathrm{MV}$ ) using robust modular components. This architecture simultaneously resolves problems found in conventional pulsed and linear induction accelerators. A variety of highbrightness pulsed $x$-ray radiographic sources are needed from sub-megavolt to $16-\mathrm{MeV}$ endpoints with greater source brightness (dose/spot ${ }^{2}$ ) than presently available. We are applying IVA systems to produce very intense (up.to $75 \mathrm{TW} / \mathrm{cm}^{2}$ ) electron beams for these flash radiographic applications.

The accelerator electromagnetic pulse is converted to a directed electron beam at the end of a selfmagnetically insulated vacuum transmission line. The cantilevered cathode threading the accelerator cavities terminates in a small (1-mm diameter) needle, producing the electron beam which is transported to a grounded bremsstrahlung converter within a strong $(-50-T)$ axial magnetic field. These systems produce $\mathrm{mm}$-sized stable electron beams, yielding very intense $\mathrm{x}$-ray sources.

Detailed simulations of the electron beam generation, transport, and target interaction are presented along with scaling laws for the radiation production and $x$-ray spot size. Experimental studies confirm these simulations and show this reliable, compact, and inexpensive technology scales to $1000-R$ doses a meter from a mm-diameter source in $50 \mathrm{~ns}$.

\section{INDUCTIVE VOLTAGE ADDER}

The Inductive Voltage Adder architecture was first demonstrated on the Helia [1] accelerator in 1985, and subsequently has been used in HERMES [2], Sabre [3], Reiden-IV [4], RHEPP [5], the DARHT injector [6], and Kalif-Helia [7]. Most recently we have used this approach in the Radiographic Integrated Test Stand (RITS) [8]. The IVA architecture allows power pulse shaping and switching at the well understood and reliable few million volt and few ohm levels, with subsequent voltage addition through ferromagneticisolated stages to the terminal voltage.

Figure 1 shows the RITS architecture. A fast Marx charges two water dielectric transfer capacitors to 3 $\mathrm{MV}$ in a $\mu \mathrm{s}$. Laser triggered gas switches couple each capacitor to three, 7.8-ohm, 60-ns water pulse forming lines (PFLs) which drive, through self-closing switches, matched impedance and length output transmission lines (Fig. 2). A second water peaking switch then transfers the pulse into a third matched

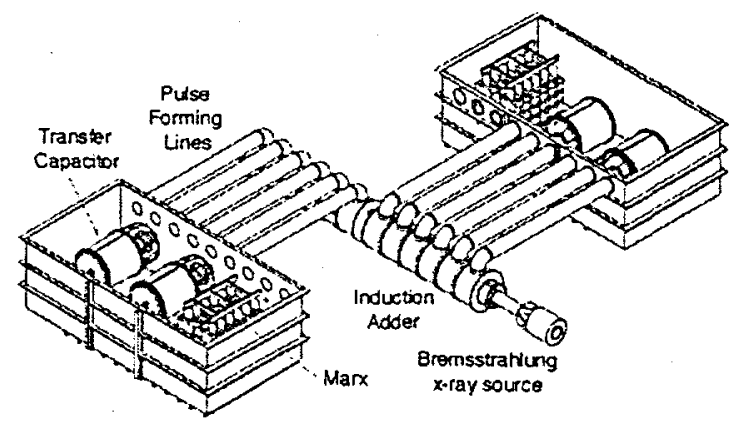

Figure 1. Radiographic Integrated Test Stand Inductive Voltage Adder accelerator. The 16-MV configuration shown is designed to provide 1000 rads a meter from a 1 -mm radiographic source

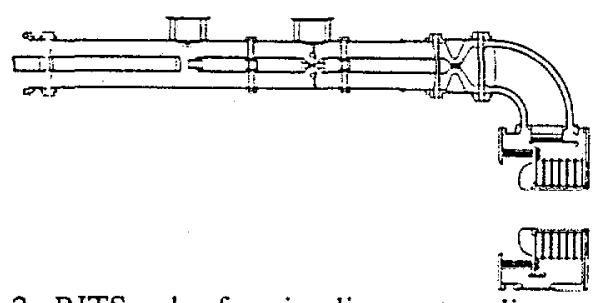

Figure 2. RITS pulse forming line, output lines, and induction cavity. The system is designed for wave shape control and stability with fast rise-times and minimal pre-pulse.

\footnotetext{
- Sandia is a multiprogram laboratory operated by Sandia Corporation, a Lockheed Martin Company, for the United States Department of Energy under Contract DE-ACO4-94AL85000.
} 


\section{DISCLAIMER}

This report was prepared as an account of work sponsored by an agency of the United States Government. Neither the United States Government nor any agency thereof, nor any of their employees, make any warranty, express or implied, or assumes any legal liability or responsibility for the accuracy, completeness, or usefulness of any information, apparatus, product, or process disclosed, or represents that its use would not infringe privately owned rights. Reference herein to any specific commercial product, process, or service by trade name, trademark, manufacturer, or otherwise does not necessarily constitute or imply its endorsement, recommendation, or favoring by the United States Government or any agency thereof. The views and opinions of authors expressed herein do not necessarily state or reflect those of the United States Government or any agency thereof. 


\section{DISCLAIMER}

Portions of this document may be illegible in electronic image products. Images are produced from the best available original document. 
water transmission line which, through an oil switch, drives an induction cavity. The sequential PFLs assure accurate pulse shape control and pre-pulse minimization for $95 \%$ of all shots, as shown in Fig. 3.

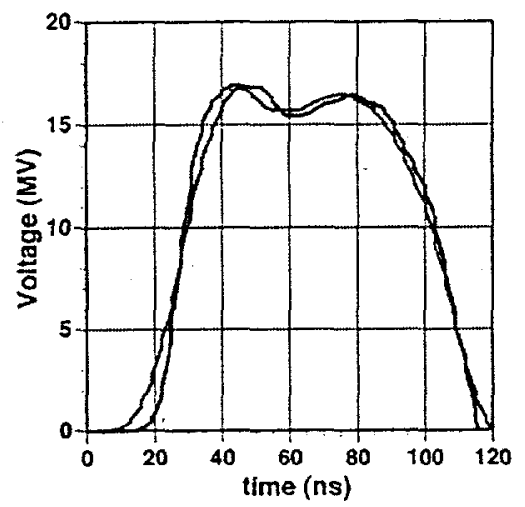

Figure 3. RITS output pulse variation. Statistical analysis shows that $95 \%$ of all shots will lie between blue (nominal) and green curves. The principal difference is an increase in rise time from 13 to $21 \mathrm{~ns}$.

The induction cavities incorporate a unique tapered azimuthal transmission line to uniformly load the vacuum insulators and provide a symmetric radial wave to the adder. These series cavities are threaded by a grounded cathode stalk, forming a coaxial vacuum transmission line whose impedance grows through the adder to match the injected wave impedance. The output transmission line dimensions are chosen to operate in a magnetically inhibited flow, where a fraction of the current is carried in an ExB drifting cathode sheath [9].

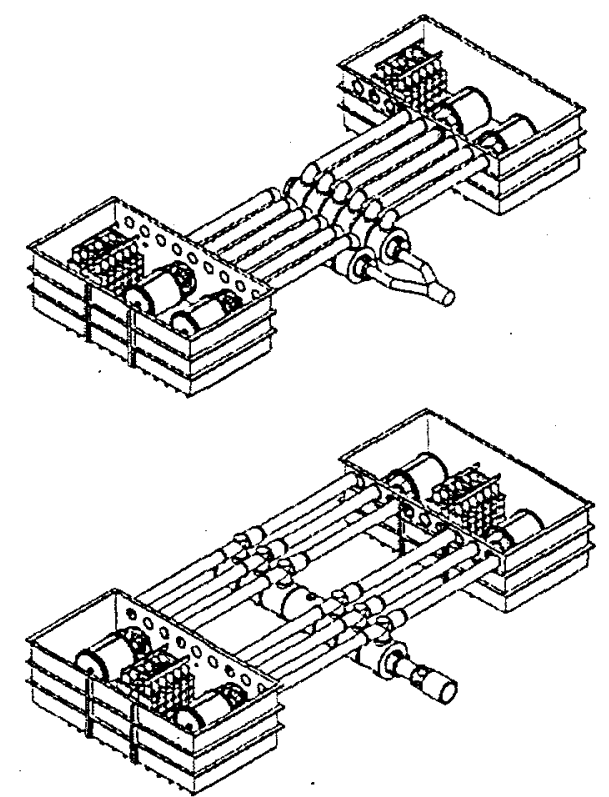

Figure 4. Two pulse $8 \mathrm{MV}$ RITS configurations: top: independent accelerators driving sequentialadjacent-pulse sources within the same diode; bottom: independent accelerators coupled through induction cavities multi-pulsing a single $\mathrm{x}$-ray source.
RITS will be assembled in two identical 6-cavity modules, each capable of providing a single 50 -ns (full width at $90 \%$ maximum), 8-MV, 50-ohm pulse. These modules are designed to be operated in a variety of configurations: in series (16 MV, 1000 rads a meter from a $1-\mathrm{mm}$ source spot as shown in Fig. 1); separately, driving close proximity radiographic sources (few mm spacing in a sequential-adjacent-pulse mode); or in parallel (multi-pulsing a single MITL) (Fig. 4). Thus RITS will support the entire suite of single- and double-pulse pulsed-power driven flash radiographic technology demonstrations.

\section{MAGNETICALLY IMMERSED ELECTRON DIODE}

High-brightness flash radiography requires coupling the coldest possible, highest intensity electron beam into the smallest area of a high-atomic number anode at a voltage appropriate to radiograph the object under investigation. Pinched beam and paraxial diodes have historically been used on a spectrum of pulsed power drivers coupling up to $30 \mathrm{kA}$ into $3-\mathrm{mm}$ diameter spots [10]. Magnetically immersed diodes have also been extensively studied [11], and are now being applied to this problem with the goal of coupling $50 \mathrm{kA}$ into a $1-\mathrm{mm}$ diameter spot [12].

Figure 5 shows the magnetically immersed electron beam diode geometry. The IVA cathode cantilever extends beyond the final cavity to the experimental test area, tapering to a $0.5-\mathrm{mm}$ diameter. The surrounding coaxial vacuum enclosure terminates in a pulsed solenoid magnet. MITL sheath electrons unable to enter the solenoid loss cone are shunted to the wall, while a cold beam is drawn from the needle boundary current and is transported in the axial

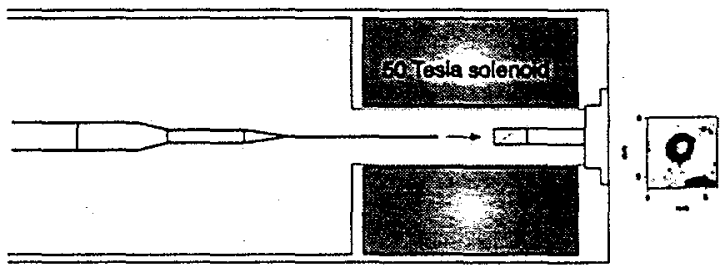

Figure 5. Magnetically immersed diode. MITL sheath electrons are diverted at the magnet entrance while a cold high intensity beam is drawn from the boundary current in the cathode needle. Inset: the measured HERMES $x$-ray spot from this diode.

magnetic field to the bremsstrahlung converter target. Extensive analytic and numerical modeling [13] suggests the electron current can be simply described by the space charge limit

$$
I_{\text {beam }}=\frac{17 k A\left(1+\frac{V}{511 k V}\right)}{\left(1-\frac{Z n_{i}}{n_{e}}\right)\left(1+2 \ln \left(R_{\text {wall }} / r_{\text {beam }}\right)\right)}
$$


and the $\mathrm{x}$-ray source size is just the needle size added in quadrature to the electron gyro-orbit in the applied magnetic field (Figs. 6, 7):

$s p o t=2.6 \sqrt{r_{c}^{2}+\left(\frac{0.17 \text { Tesla }}{B_{z}} \sqrt{2 \gamma / 17 k A}\right)^{2}}$

The particle in cell simulations shown in Fig. 7 predict electron and ion motion during the pulse and are used to optimize the transition and immersed diode regions (Fig. 7). It is found that balancing the electric and magnetic profiles in the presence of the evolving beam and wall particle flows is critical to designing a highly focused radiographic source(Fig. 8).

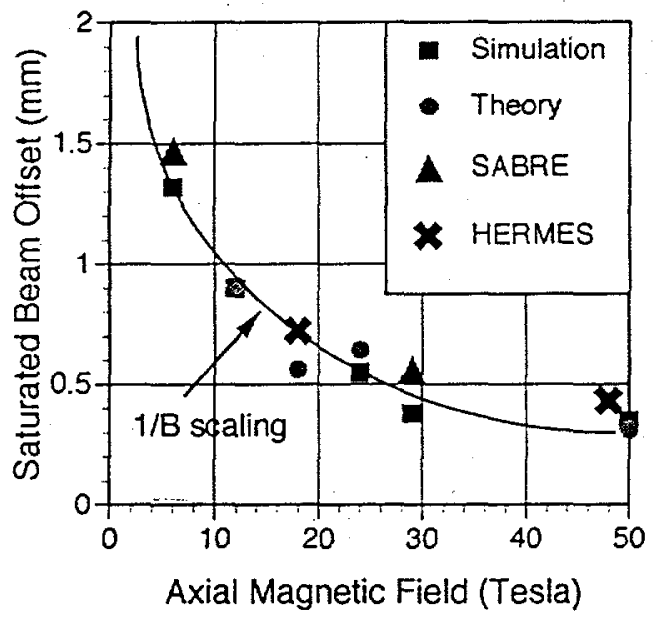

Figure 6. Beam spot size scaling. The simple analytic model agrees well with both PIC simulations and experimental data from two accelerators.

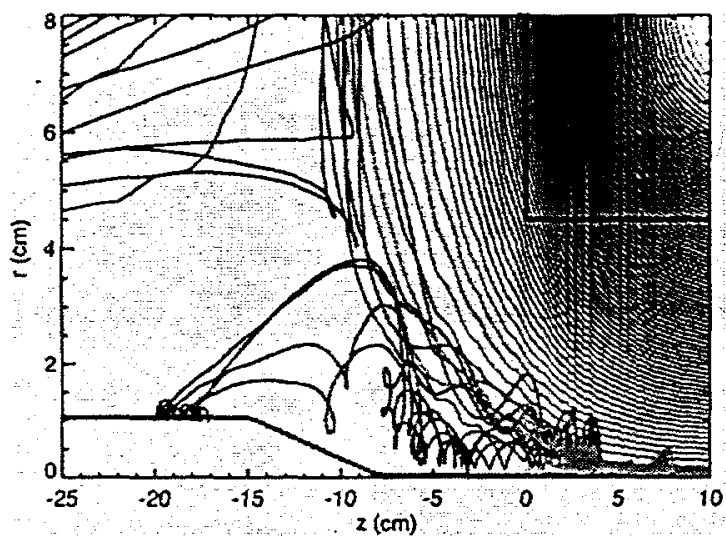

Figure 7. 2D and 3D PIC simulations enable optimized transition and diode designs. The electron trajectories show paths from different emission locations in the applied magnetic field and can be tallied to differentiate spot and halo sources.

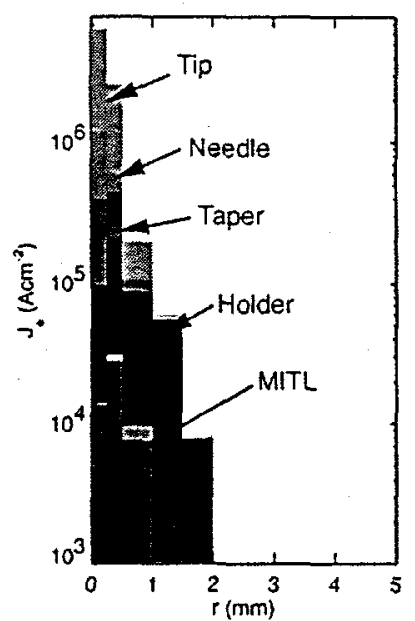

Figure 8. Calculated electron distribution on target, tallied by emission site.

\section{RADIOGRAPHIC SOURCE}

Monte Carlo calculations have been used to model the $x$-ray production from these intense electron beam sources interacting with high atomic number (typically Tungsten or Tantalum) anodes. The PIC electron trajectories discussed in the previous section were used as the source distribution into the Integrated Tiger Series [14] Monte Carlo model of the experimental geometry, and the results compared to experimental data. Since $x$-ray source spatial distribution and dose production are the experimental measurables, it is important to relate them to the incident (PIC) electron distribution. Figure 9 displays this comparison, showing that the measured $x$-ray spot size can be directly linked to the electron source distribution for this class of diodes. This correlation has been used to display the experimental data in Fig. 6.

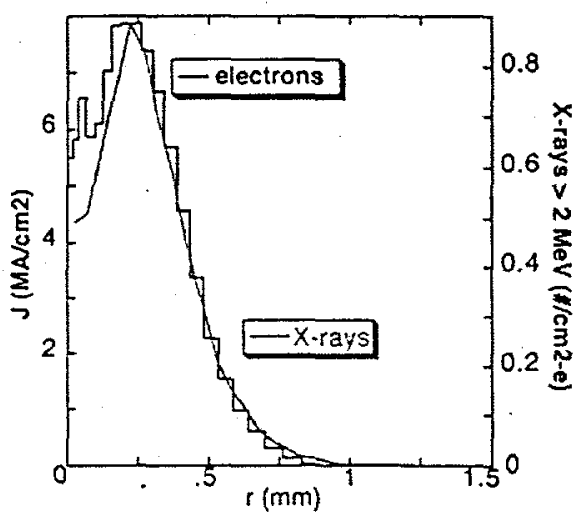

Figure 9. Correlation between electron source and $x$ ray profiles. The $x$-ray spot can be used as indicative of the electron beam distribution at the target.

Figure 10 shows the results for a suite of such calculations above $6 \mathrm{MV}$, where the circles represent HERMES experimental data [15]. The shaded region 
represents the parameter space capable of producing $>$ 1000 rads a meter from the $x$-ray source for the tested diode. All the experimental data lie on this surface, confirming an $\mathrm{x}$-ray production scaling of:

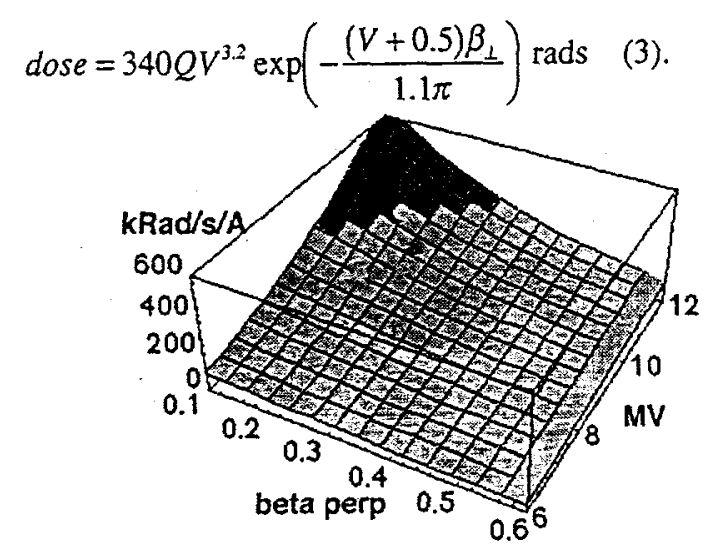

Figure 10. Monte Carlo x-ray production predictions for the HERMES magnetically immersed diode. Experimental data shown confirms the model shape and the generation of a cold focused electron beam.

\section{REFERENCES}

[1] J.J. Ramirez et al., "The Four Stage Helia Experiment," ' $5^{\text {th }}$ IEEE Pulsed Power Conference, Arlington, Virginia, June 10-12, 1985.

[2] J.J. Ramirez et al., "The HERMES-III Program," Proceedings of the $6^{\text {th }}$ IEEE Pulsed Power Conference, Arlington Virginia, June 29 - July 1, 1987.

[3] J.P. Corley et al., "SABRE, A 10-MV Linear Induction Accelerator," Proceedings of the $8^{\text {th }}$ International Pulsed Power Conference, San Diego, California, June 16-19, 1991.

[4] T. Akiba et al., "Development of electron injector with induction units," Proceedings of the $6^{\text {th }}$ International Conference on High-Power Particle Beams, Kobe, Japan, June 9-12, 1986.

[5] D.L. Johnson et al., "Results of Initial Testing of the Four Stage RHEPP Accelerator," Proceedings of the $9^{\text {th }}$ International Pulsed Power Conference, Albuquerque, New Mexico, June 21-23, 1993.

[6] R.L. Carlson et al., "A 4-MV, 4-kA Four Pulse Injector for DARHT Axis 2," this conference.

[7] J. Fockler et al., "Design of a $6 \mathrm{MV}, 2 \mathrm{TW}$ Positive Ion Driver using an Induction Adder," $g^{\text {th }}$ International Pulsed Power Conference, Albuquerque, New Mexico, June 21-23, 1993.

\section{FUTURE PLANS}

Having demonstrated the concept of IVA accelerators powering magnetically immersed electron diodes as radiographic $x$-ray sources on the Sabre and HERMES facilities, we are scaling this source to both higher and lower voltages with the construction of RITS and TriMeV [16]. RITS will be the first IVA driver optimized for this specific application, and will test a number of pulsed power technologies and approaches needed for the future as well as provide the basis for confidence in high-brightness multi-pulse pulsed power radiographic technology. While RITS is under construction, TriMeV is being utilized in Nevada to develop further understanding of the different possible radiographic sources (pinched beam, paraxial, rod pinch, and immersed diodes) to provide a basis for application specific technology decisions.

[8] Ian Smith, "Design of a Radiographic Integrated Test Stand (RITS) based on a voltage adder, to drive a diode immersed in a high magnetic field," Paper O13AB, this conference.

[9] J. Creedon, Journal of Applied Physics 46, 2946, 1975.

[10] D.W. Forster, "Optimization of Modular Pulsed Power Machines," AWE/HD/B/9701/R01, January-1997.

[11] L.M. Friedman and M. Ury, Rev. Sci. Instrum. $41,1334,1970$.

[12] M.G. Mazarakis et. al. Appl. Phys. Lett. 70, 832, 1996.

[13] D. Welch and T. Hughes, Laser and Particle Beams 16 (2), 285, 1998.

[14] J.A. Halbleib, R.P. Kensek, T.A. Mehlhorn, G.D. Valdez, S.M. Setzer, and M.J. Berger, IEEE Tran. Nuc. Sci. 39, 1025 (1992).

[15] Peter Menge, "Experimental time resolved electron beam temperature measurements using bremsstrahlung diagnostics," paper PB084, this conference.

[16] Eugene Hunt et al., "The TriMeV Accelerator for Pulsed Power Driven Radiography Experiments," $26^{\text {th }}$ International Conference on Plasma Science, Monterey, California, June 20-24, 1999. 